e. $S_{\text {cripła }} \mathbf{R}_{\text {omanica }}$

vol. 6 (2018): 1-16

\title{
Secciones Bilingües de español en el extranjero: estudio sobre la motivación y actitudes del alumnado en el aprendizaje del español como lengua extranjera
}

\author{
Bilingual Sections of Spanish abroad: study on the motivation and \\ attitudes of students in learning Spanish as a foreign language
}

María del Mar Manzano Barquilla

Universidad de Cádiz, España

\begin{abstract}
Resumen: El objetivo de este trabajo es analizar el perfil motivador y actitudinal del alumnado de español como lengua extranjera en una Sección Bilingüe de español en el extranjero. En concreto, se ha realizado el estudio en la sección bilingüe del Liceum XXXII Halina Poświatowska, ubicado en la ciudad de Łódź (Polonia) y dependiente de los Ministerios de Educación español y polaco. En el documento se introduce dicho programa de cooperación educativa en el exterior, se presenta la literatura sobre las variables afectivas en el aprendizaje de una lengua extranjera y la metodología de investigación.
\end{abstract}

Palabras clave: educación bilingüe, motivación, actitudes, aprendizaje de lenguas extranjeras, secciones internacionales.

Abstract: The aim of this paper is to analyse the motivation and attitudes in learning Spanish as a Foreign Language in an International Section of Spanish Language. This study has been carried out specifically in the bilingual section of Liceum XXXII Halina Poświatowska, which is located in Łódź (Poland) and depends on Spanish and Polish Ministries of Education. This article introduces this program of overseas educational cooperation, presents the affective variables in second language learning and research methodology.

Keywords: bilingual education, motivation, attitudes, second language learning, international sections

\section{Introducción}

El interés de este estudio descriptivo de encuesta reside en el deseo de identificar qué tipos de motivación predominan en el alumnado de la sección internacional de español en el Liceum XXXII Halina Poświatowska, así como el grado de motivación. Para describir la motivación del alumnado de español como lengua extranjera (ELE), partimos de la definición de Madrid (1999):

Un estado interno del individuo influenciado por determinadas necesidades y/o creencias que le generan actitudes e intereses favorables hacia una meta, y un deseo que le mueve a conseguirla con dedicación y esfuerzo continuado (p. 69). 
Considerando que la motivación es el resultado de la fusión del deseo por aprender y del esfuerzo que se realiza para ello (Gardner, 1985), el análisis comienza por el grado de motivación, queriendo así determinar la intensidad (deseo + interés + esfuerzo) de la misma, como paso previo a la identificación de los tipos de motivación. Para ello, se han seguido las dicotomías de Tragant y Muñoz (2000)', que proponen las categorías de motivación intrínseca y extrínseca, dividiendo esta segunda en dos subcategorías, a saber, instrumental e integradora². Esta clasificación permite delimitar las variables en función de la razón que empuja al aprendizaje, a la vez que establecer el tipo de motivación dominante.

El concepto de motivación resulta de un constructo multidimensional, cuyo estudio, de acuerdo con Ezpí y Azurmendi (1996), queda incompleto si no atendemos a las actitudes, entendidas como la predisposición hacia algo o la postura hacia alguien, pues juegan un papel en el origen y/o en las consecuencias de la motivación.

Por tanto, con el fin de alcanzar una visión de esta realidad lo más global posible, se ha optado por detectar las actitudes del alumnado, tanto hacia distintos factores del proceso de enseñanza aprendizaje, como hacia los factores socioculturales de la comunidad objeto, siguiendo, en primer lugar, el modelo propuesto por Dörnyei (1994) en el que destaca el contexto ambiental o factores situacionales ${ }^{3}$. De esta forma, se han establecido como variables la actitud hacia la situación de aprendizaje, desglosada en actitud hacia el docente, actitud hacia el grupo-clase, actitud hacia el entorno de aprendizaje (recursos materiales, mobiliario, etc.) y actitud hacia las asignaturas de español; la actitud hacia sí mismo; la actitud hacia las lenguas extranjeras y la actitud hacia lo hispano, más concretamente, hacia la lengua española, hacia la cultura hispana y hacia los hispanohablantes.

Entre todas las variables que intervienen en el proceso de enseñanza-aprendizaje de una lengua extranjera, diferentes autores han destacado la importancia de las variables afectivas (Gardner y Lambert, 1972; Spolsky, 1989; Dörnyei, 1994). En el contexto polaco, Spychała-Wawrzyniak y Sagermann (2016) realizan a finales de octubre de 2015, el primer estudio en escuelas polacas de enseñanza secundaria sobre los motivos que incitaron al alumnado de enseñanza secundaria a elegir el español como como primera y segunda lengua extranjera, destacando como resultado la motivación intrínseca. Meses antes, en junio del mismo año, se llevó a cabo la recogida de datos del presente estudio, la primera investigación en el contexto de una sección internacional de español que busca determinar el grado y los tipos de motivación, así como las actitudes del alumnado que cursa una sección bilingüe de español.

Dichas secciones bilingües de español ${ }^{4}$ en el extranjero son fruto de programas de cooperación educativa entre el Ministerio de Educación español y sus homólogos en diferentes Estados, con el fin de difundir la lengua y culturas

\footnotetext{
1 p. 82

2 Intrínseca: deseo que se activa internamente en ausencia de consecuencias. Extrínseca: surge de estímulos externos. Instrumental (extrínseca): medio para conseguir otros fines. Integradora (extrínseca): propósito utilitario. Tiene un carácter sociocultural ya que busca la integración o comprensión de una comunidad.

3 Del clima o marco del aula se desprenden la actitud hacia la situación de aprendizaje.

4 Principal acción educativa del Estado español en Polonia.
} 
españolas en el extranjero. De esta forma, se ofertan programas bilingües de español en centros educativos públicos de enseñanza secundaria en China, Turquía, y algunos países participantes de Europa (Rusia, Rumania, República Checa, Polonia, Hungría, Eslovaquia y Bulgaria). El Real Decreto 1027/1992, de 25 de junio, regula en el Estado español la acción educativa en el exterior. Dicha acción educativa se concreta en Polonia en la existencia de 14 Secciones Bilingües de español que vienen funcionando desde septiembre de 2007. En ellas, se imparten tres cursos académicos correspondientes al Bachillerato en el que el currículo polaco es complementado por algunas materias impartidas en español: ELE, Lengua y Literatura ampliadas y Cultura (Geografía e historia, Historia de España e Historia del Arte español). Al final del programa los alumnos tienen opción de obtener tanto el Título de Bachiller polaco como el español.

Queriendo conocer cuál es la motivación del alumnado que opta por escolarizase en estas secciones y sus actitudes en el aprendizaje del español como lengua extranjera, se ha llevado a cabo un estudio en la Sección Bilingüe de español situada en el Liceum XXXII Halina Poświatowska de la ciudad de Łódź. Para ello, se ha hecho uso del cuestionario Minera Reyna (2010) que, adaptado a este contexto 5 , permite trazar el perfil motivador y actitudinal en el aprendizaje de una lengua extranjera.

\section{Introducción}

\subsection{Población objeto de estudio y muestra}

La población objeto de estudio está conformada por el alumnado de la sección bilingüe de español del Liceum número XXXII Halina Poświatowska de la ciudad de Łódź, la tercera ciudad más poblada de Polonia, tras Varsovia y Cracovia, respectivamente.

De los cuatro cursos que conforman la sección bilingüe de español, el cuestionario fue distribuido a los tres primeros cursos $(\mathrm{OH}, 1 \mathrm{H}$ y $2 \mathrm{H})$ ya que el último curso académico $(3 \mathrm{H})$ había terminado con anterioridad debido a la preparación previa de las pruebas de la Matura (homóloga de la selectividad).

Siendo voluntaria la cumplimentación del cuestionario, el número de alumnos de cada grupo que participó en el estudio fue el total de aquellos que se encontraban presentes en el momento de la recogida de datos, lo que se considera un muestreo errático.

La recogida de datos se efectuó entre finales de mayo y primeros de junio de 2015. Participaron 61 informantes (54 alumnas y 7 alumnos) de tres de los cuatro cursos de la sección internacional de español del Liceum XXXII de la ciudad de Łódź. La distribución de la muestra se refleja en la tabla 1, siguiendo parámetros tales como curso y lengua materna.

\footnotetext{
5 Apdo. Il: ítem 2 abreviado, en el ítem 3 se sustituye la referencia a la universidad por Bachillerato. Apdo. V: se suprime el ítem 6 por no ser pertinente en este contexto y se sustituyen las referencias al alemán por la lengua materna del alumnado (polaco) en los ítems 5 y 8 . Apdo. VI: se suprime el ítem sobre el mundo hispano, por verse este contemplado en los ítems sobre la cultura hispana.
} 


\begin{tabular}{c|l|l|l}
\hline CURSO & $\begin{array}{l}\text { LENGUA } \\
\text { MATERNA }\end{array}$ & MUESTRA & POBLACIÓN \\
\hline $\mathbf{O H}$ & $\begin{array}{l}\text { Polaco } \\
100 \%\end{array}$ & 20 & 30 alumnos \\
\hline $\mathbf{1 H}$ & $\begin{array}{l}\text { Polaco } \\
100 \%\end{array}$ & 28 & 30 alumnos \\
\hline $\mathbf{2 H}$ & $\begin{array}{l}\text { Polaco } \\
100 \%\end{array}$ & 13 & 20 alumnos \\
\hline $\mathbf{3 H}$ & $\begin{array}{l}\text { Polaco } \\
100 \%\end{array}$ & 0 & 28 alumnos \\
\hline
\end{tabular}

Tabla 1. Distribución de la muestra

\subsection{Instrumento para la obtención de datos: el cuestionario}

En la bibliografía sobre la influencia de las variables afectivas en el aprendizaje de una lengua extranjera encontramos el cuestionario Motivación y Actitudes en el Aprendizaje de Lenguas Extranjeras (MAALE) de Minera Reyna (2010) que se adecuaba a los objetivos de este estudio y pudo ser adaptado al contexto.

La administración del cuestionario fue realizada personalmente y de forma presencial en el centro Liceum XXXII de Łódź y en las aulas habituales para la impartición de la docencia. De esta forma, obtuvimos un alto índice de respuestas ya que, del total de 108 alumnos que cursaban los tres primeros cursos de la sección internacional de español, 61 cuestionarios fueron cumplimentados, lo que supone una participación del 56,48\%. Antes de la aplicación del cuestionario, los informantes fueron informados de los objetivos de esta investigación y se les garantizó el anonimato.

El cuestionario viene introducido por un apartado para obtener información personal de los encuestados (año de nacimiento, sexo, nacionalidad, curso, lengua materna y conocimiento de lenguas extranjeras). La estructura del cuestionario está desarrollada a partir de escalas de tipo Likert y escalas de diferencial semántico con el fin de medir catorce variables que se ordenan en una división de seis apartados. En el primero de ellos se recaban datos sobre los tipos de motivación y la motivación dominante. En el segundo, acerca del grado del motivación. En los cuatro siguientes se encuentran los ítems relativos a la variables actitudinales: las actitudes hacia sí mismo, las actitudes hacia la situación de aprendizaje, las actitudes hacia la lengua extranjera y las actitudes hacia lo hispano, en los apartados 3, 4, 5 y 6 respectivamente. Para la codificación de las respuestas se utiliza el baremo siguiente:

- En los apartados 1 y 6.3, al alumnado se le presentaron enunciados acompañados de cinco respuestas graduales: "mucho" (5), "bastante" (4), "regular" (3), "poco" (2), "nada" (1). 
- El parámetro valorativo en los apartados 2, 3, 4.1 y 5 comprende de "estoy totalmente de acuerdo" (5), "estoy de acuerdo" (4), "estoy indeciso" (3), "estoy en desacuerdo" (2) a "estoy totalmente en desacuerdo" (1). En los apartados 3, 4.1 y 5 esta asignación numérica se invierte cuando los enunciados son formulados negativamente. Esta es una práctica habitual para evitar las respuestas sistemáticas.

- En el apartado 4.4 las opciones oscilan de "muy bueno" (5), "bueno" (4), regular (3), malo (2) a muy malo (1).

- En los apartados 4.2, 4.3, 6.1 y 6.2 se emplean escalas de diferencial semántico con seis posibilidades de elección. El valor numérico asignado a los pares de adjetivos polares se mueve del 1 en los adjetivos negativos al 6 en los adjetivos positivos. De nuevo se intercala el orden, en este caso adjetivos negativos y positivos, para evitar las respuestas sistemáticas.

\section{Procedimiento de análisis de datos}

Arnal, Del Rincón y Latorre (1992) establecen diferentes tipos de variables según el nivel de medición. En base a esto, se pueden clasificar las variables estudiadas en el presente cuestionario como de tipo cualitativa ordinalk.

Tras la codificación de las respuestas marcadas por el alumnado a cada uno de los ítems, se realizan el análisis de la media obtenida del grupo respecto a las variables y la moda como medidas de tendencia central y la desviación típica como medida de dispersión. Asimismo, se presentan las distribuciones porcentuales de respuesta gráficamente en diagramas de secciones. Se opta por los diagramas de secciones como forma de presentación de resultados de frecuencia puesto que permiten mostrar de manera visual el comportamiento de los informantes.

Para el estudio de las posibles correlaciones entre los factores motivacionales y actitudinales se ha aplicado el Coeficiente de Correlación de Pearson (r), cuyo valor de índice puede variar en el intervalo de -1 (señalando una relación inversa) a +1 (indicando una relación directa) entre las variables. Se ha realizado la correlación entre las catorce variables del estudio, considerando los valores de referencia de correlación directa para investigaciones lingüísticas propuestos por Cohen (1977) y revalidados por Bachman (2004).

\section{Resultados}

A continuación, se muestran los resultados obtenidos en el estudio conforme a las variables de la investigación. En cada uno de los apartados del análisis estadístico se presentan tanto la puntación máxima que es posible obtener en cada uno de ellos, como la puntuación media alcanzada en el total del alumnado de los tres grupos de la sección internacional tomados como unidad, lo cual facilita una visión global del grupo de informantes.

\subsection{Resultados en relación a los ítems sobre las variables motivacionales}

En primer lugar, se buscaba identificar qué tipos de motivación predominan en el alumnado de la sección internacional de español en el Liceum XXXIl de Łódź y, además, comprobar el grado de motivación.

\footnotetext{
${ }^{6}$ La variable puede tomar distintos valores siguiendo una escala ordenada.
} 


\subsubsection{Apartado 1, sobre los tipos de motivación y la motivación dominante}

El valor mínimo que es posible obtener en cada tipo de motivación es de 61 y el valor máximo es de 1220 puntos (61 informantes por el producto de un máximo de 20 puntos cada uno). En el siguiente diagrama observamos el comportamiento de dominancia de la motivación intrínseca, que ha puntuado un total de 1056 puntos, seguida de la instrumental (951 puntos) y la integradora (901).

\section{Tipos de Motivación}

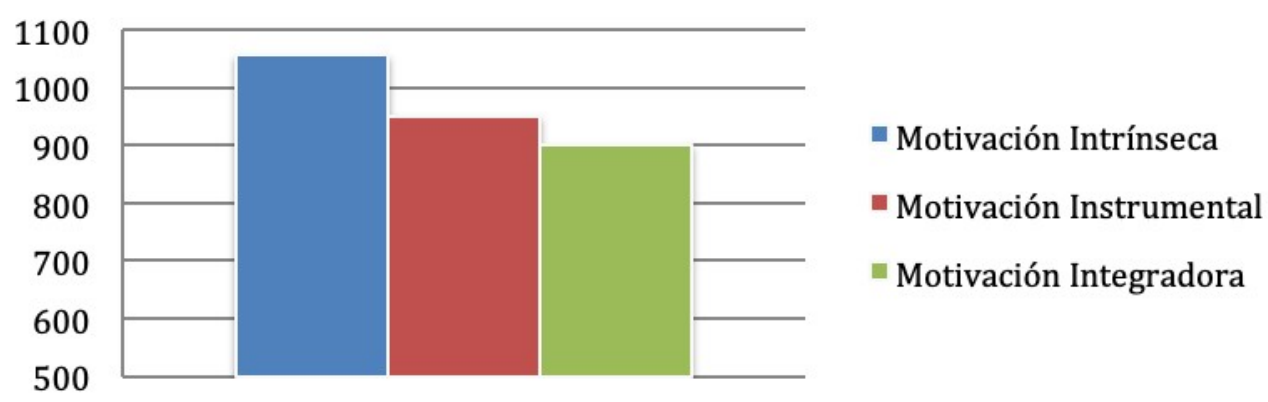

Gráfica 1: Media de la motivación dominante

Siguiendo el baremo empleado7, las medias presentadas en la tabla 2 permiten enmarcar el grado de motivación intrínseca como muy alto, y los grados de las motivaciones instrumental e integradora como altos.

\begin{tabular}{|l|l|l|l|l|}
\hline $\begin{array}{l}\text { Tipo de } \\
\text { Motivación }\end{array}$ & $\begin{array}{l}\text { Puntuación } \\
\text { Máxima }\end{array}$ & $\begin{array}{l}\text { Puntuación } \\
\text { Obtenida }\end{array}$ & Media & $\begin{array}{l}\text { Desviación } \\
\text { Típica }\end{array}$ \\
\hline Intrínseca & 1220 & 1056 & 17,31 & 2,26 \\
\hline Instrumental & 1220 & 951 & 15,59 & 3,17 \\
\hline Integradora & 1220 & 901 & 14,77 & 3,14 \\
\hline
\end{tabular}

Tabla 2: Motivación dominante global

En la tabla 3 se refleja el porcentaje de informantes con una motivación dominante, considerando también el caso en el que la motivación dominante no es única, como "varias", sea cual sea la combinación. Se observa que la motivación dominante individual en el $43 \%$ de los sujetos es también la motivación intrínseca.

\footnotetext{
7 Muy alto (17-20), alto (13-16), medio (9-12), bajo (5-8) y muy bajo (1-4)
} 


\begin{tabular}{|l|l|l|}
\hline $\begin{array}{l}\text { Tipo de motivación } \\
\text { dominante }\end{array}$ & $\begin{array}{l}\text { Número de } \\
\text { informantes }\end{array}$ & Porcentaje \\
\hline Intrínseca & 26 & $43 \%$ \\
\hline Integradora & 4 & $6 \%$ \\
\hline Instrumental & 9 & $15 \%$ \\
\hline Varias & 22 & $36 \%$ \\
\hline Total & 61 & $100 \%$ \\
\hline
\end{tabular}

Tabla 3: Tipo de motivación dominante individual

En resumen, tanto los datos arrojados en el análisis global de grupo como en el análisis individual determinan que la motivación intrínseca es la dominante entre el alumnado de la sección internacional de español del Liceum XXXII.

\subsubsection{Apartado 2, sobre el grado de motivación}

Tal y como se evidencia en la tabla 4, la media del grado de motivación en cada participante es de 47,67 en una escala de 1 a 60 puntos, lo que indica un grado de motivación alto8.

\begin{tabular}{|l|l|l|l|}
\hline Puntuación Máxima & Puntuación Obtenida & Media & Desviación Típica \\
\hline 3660 & 2908 & 47,67 & 6,59 \\
\hline
\end{tabular}

Tabla 4: Grado de motivación global en los tres grupos

Cabe destacar que la mayoría de los informantes, un $93 \%$, tiene un grado de motivación entre alto y muy alto independientemente, mientras el restante $7 \%$ presenta una motivación media, como se refleja en el histograma. Igualmente, puede verse que la moda en el grado de motivación es "muy alto". Este resultado permite afirmar que alumnado en esta sección internacional de español está motivado para aprender la lengua española.

\begin{tabular}{|l|l|l|}
\hline $\begin{array}{l}\text { Grado de de } \\
\text { motivación }\end{array}$ & $\begin{array}{l}\text { Número } \\
\text { informantes }\end{array}$ & Porcentaje \\
\hline Muy alto & 29 & $47 \%$ \\
\hline Alto & 28 & $46 \%$ \\
\hline Medio & 4 & $7 \%$ \\
\hline Total & 61 & $100 \%$ \\
\hline
\end{tabular}

Tabla 5: Grado de motivación en porcentajes

\footnotetext{
8 Según el baremo, alto: 37-48.
} 


\subsection{Resultados en relación a los ítems sobre las variables actitudinales}

En segundo lugar, se han querido identificar las actitudes manifestadas por el alumnado de la sección internacional de español en el Liceum XXXII de Łódź, tanto hacia distintos factores del proceso de enseñanza aprendizaje, como hacia factores socioculturales de la comunidad objeto. Se debe señalar que no se han encontrado actitudes negativas en el grupo de informantes hacia ninguno de los aspectos estudiados.

A modo de síntesis, se presentan las medias globales y la desviación típica del total de participantes en la tabla 5 y el análisis individual en porcentajes en la tabla 6.

A continuación, se muestran las puntuaciones obtenidas en las diferentes actitudes estudiadas según el análisis global, esto es, tomando la puntuación obtenida del total de informantes:

\begin{tabular}{|l|l|l|l|l|l|}
\hline $\begin{array}{l}\text { Actitud } \\
\text { hacia }\end{array}$ & $\begin{array}{l}\text { Puntuación } \\
\text { global máxima } \\
\text { posible }\end{array}$ & $\begin{array}{l}\text { Puntuación } \\
\text { total del } \\
\text { grupo }\end{array}$ & $\begin{array}{l}\text { Puntuación } \\
\text { máxima por } \\
\text { ítem }\end{array}$ & $\begin{array}{l}\text { Media } \\
\text { del } \\
\text { grupo }\end{array}$ & Desviación \\
Típica
\end{tabular}

Tabla 6: Medias globales de las actitudes estudiadas

En cuanto al análisis individual, la distribución porcentual de la actitud del alumnado demuestra que sobresalen las actitudes positivas y excelentes. La tabla 7 
permite apreciar en negrita la moda y la concentración de la muestra hacia el extremo "excelente" y "muy buena".

\begin{tabular}{|l|l|l|l|l|l|}
\hline Actitud hacia & Excelente & Muy buena & Buena & Regular & Mala \\
\hline Sí mismo & $30 \%$ & $\mathbf{5 7 \%}$ & $13 \%$ & $0 \%$ & $0 \%$ \\
\hline El profesor & $13 \%$ & $\mathbf{6 1 \%}$ & $21 \%$ & $5 \%$ & $0 \%$ \\
\hline El grupo & $30 \%$ & $\mathbf{4 8 \%}$ & $18 \%$ & $5 \%$ & $0 \%$ \\
\hline El curso & $5 \%$ & $\mathbf{6 9 \%}$ & $21 \%$ & $3 \%$ & $2 \%$ \\
\hline El entorno de aprendizaje & $7 \%$ & $\mathbf{7 5 \%}$ & $16 \%$ & $0 \%$ & $2 \%$ \\
\hline Las lenguas extranjeras & $\mathbf{5 1 \%}$ & $44 \%$ & $5 \%$ & $0 \%$ & $0 \%$ \\
\hline El español & $\mathbf{5 4 \%}$ & $44 \%$ & $2 \%$ & $0 \%$ & $0 \%$ \\
\hline Los hispanohablantes & $\mathbf{5 6 \%}$ & $41 \%$ & $3 \%$ & $0 \%$ & $0 \%$ \\
\hline La cultura hispana & $23 \%$ & $\mathbf{5 4 \%}$ & $21 \%$ & $2 \%$ & $0 \%$ \\
\hline
\end{tabular}

Tabla 7: Distribución porcentual de las variables actitudinales

Por un lado, despuntan las actitudes hacia las lenguas extranjeras en general (51\%), hacia la lengua española (54\%) y hacia los hispanohablantes (56\%), en las que más de la mitad de los informantes muestra una actitud excelente. Por otro lado, se ha de señalar que la mayoría de los participantes tiene una actitud muy buena hacia sí mismo (57\%), hacia el profesor (61\%), hacia el grupo (48\%), hacia el curso (69\%), hacia el entorno de aprendizaje (75\%) y hacia la cultura hispana (54\%).

\subsubsection{Apartado 3, sobre la actitud hacia sí mismo}

La actitud hacia sí mismo que manifiesta la muestra de aprendientes de español, es de un promedio de 15'2 puntos, lo que supone una actitud muy buena, según el baremo aplicado en este apartado del cuestionario?.

\subsubsection{Apartado 4, sobre las actitudes hacia la situación de aprendizaje}

La actitud hacia el profesorado (20'84), la actitud hacia el grupo-clase (21,33 puntos) y la actitud hacia las asignaturas de español $(19,54)$ reveladas por los informantes se encuadran en el rango de actitudes muy buenas ${ }^{10}$. La actitud hacia el entorno de aprendizaje (libro, número de participantes, material didáctico, mobiliario y aula) también alcanza la categoría de muy buena, con 17,18 puntos ${ }^{11}$.

\footnotetext{
9 Excelente (17- 20), muy buena (13-16), buena (9-12), regular (5-8), mala (1-4).

10 Según el baremo aplicado (excelente: 25- 30); (muy buena 19-24); (buena 13-18); (regular 7-12); (mala 1-6).

${ }^{11}$ Excelente (21-25), muy buena (16-20), buena (11-15), regular (6-10), mala (1-5).
} 


\subsubsection{Apartado 5, sobra la actitud hacia las lenguas extranjeras en general}

Las categorías del baremo de este apartado del cuestionario'2 sugieren que la actitud hacia las lenguas extranjeras en general $(35,98)$ se considera muy buena, aunque roza el rango de excelente.

\subsubsection{Apartado 6, sobre la actitud hacia lo hispano}

Cabe destacar la trascendencia de las actitudes hacia los hispanohablantes $(20,25)$ y hacia la lengua española $(20,44)$ que sobresalen como excelentes ${ }^{13}$. Muy cerca le sigue la actitud del alumnado hacia la cultura hispana $(37,07)$ como muy buena ${ }^{14}$.

\subsection{Estudio de las posibles correlaciones entre las variables}

Por último, se han tratado de determinar las posibles correlaciones entre las variables afectivas estudiadas mediante la aplicación del Coeficiente de Correlación de Pearson (r) ${ }^{15}$. Las correlaciones observadas entre las variables estudiadas se detallan en la tabla 8 , donde se sombrean las puntuaciones a partir de 0,50 por alcanzar el rango de correlación ${ }^{16}$ muy alta.

\footnotetext{
12 Baremo aplicado en ese apartado: excelente (37 - 45), muy buena $(28-36)$, buena (19 - 27), regular (10-18), mala (1-9).

13 Baremo aplicado en ese apartado: excelente $(20-24)$, muy buena $(15-19)$, buena $(10-14)$, regular (5-9), mala (1-4).

${ }^{14}$ Baremo aplicado en ese apartado: excelente $(41-50)$, muy buena $(31-40)$, buena $(21-30)$, regular $(11-20)$, mala (1-10).

15 Solo puede establecer relaciones lineales, no implica una relación de causalidad, ni excluye la existencia de relaciones no lineales.

${ }^{16} \mathrm{El}$ análisis de la correlación se ha basado en los valores de referencia para investigaciones lingüísticas propuestos por Cohen (1977) y revalidados por Bachman (2004).
} 


\begin{tabular}{|c|c|c|c|c|c|c|c|c|c|c|c|c|c|}
\hline 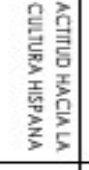 & 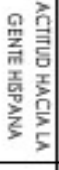 & 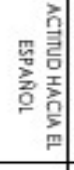 & 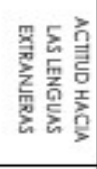 & 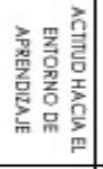 & 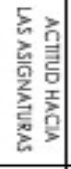 & 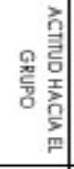 & 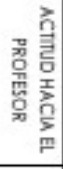 & 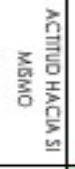 & 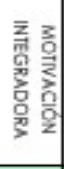 & 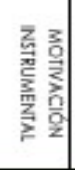 & 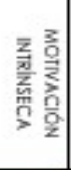 & $=2$ & \\
\hline$\stackrel{\circ}{9}$ & $\stackrel{\circ}{\mathrm{g}}$ & 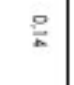 & 음 & 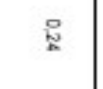 & 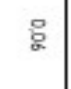 & 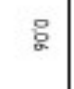 & $\stackrel{\partial}{\partial}$ & 善 & $\stackrel{g}{\sharp}$ & $\stackrel{\circ}{\circ}$ & $\stackrel{\circ}{\circ}$ & 곡 & 产 \\
\hline 咅 & 言 & 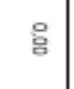 & 흠 & 웄 & $\stackrel{0}{\circ}$ & $\frac{\partial}{\Delta}$ & 音 & $\stackrel{\circ}{*}$ & : & $\stackrel{\circ}{\circ}$ & $\underline{z}$ & & 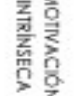 \\
\hline 형 & 8 & 뭉 & 믐 & $\frac{0}{\sigma}$ & 잉 & 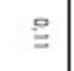 & 흠 & 8 & 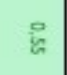 & $\stackrel{z}{\gg}$ & & & 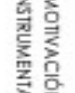 \\
\hline 吕 & : & 음 & 영 & $\stackrel{0}{\xi}$ & 홈 & $\stackrel{\circ}{\because}$ & : & 일 & $\frac{z}{>}$ & & & & \\
\hline 总 & $\frac{\circ}{90}$ & $\stackrel{\circ}{=}$ & $\stackrel{\square}{\square}$ & $\stackrel{\circ}{\Xi}$ & $\stackrel{\circ}{\circ}$ & 足 & 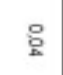 & $\frac{z}{3}$ & & & & & \\
\hline 음 & 总 & 总 & 홍 & 总 & 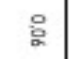 & 홈 & $z$ & & & & & & \\
\hline$\stackrel{ }{\circ}$ & 옳 & $\stackrel{\circ}{\circ}$ & 总 & $\stackrel{\circ}{3}$ & 总 & $\frac{z}{s}$ & & & & & & & \\
\hline 음 & $\frac{0}{0}$ & $\stackrel{\circ}{=}$ & : & $\frac{\partial}{\partial}$ & $\frac{z}{y}$ & & & & & & & & \\
\hline 읍 & 흠 & $\stackrel{\circ}{\circ}$ & 8 & $\frac{z}{x}$ & & & & & & & & & \\
\hline 몽 & 몽 & 욥 & $\frac{z}{x}$ & & & & & & & & & & \\
\hline$\stackrel{乛}{Z}$ & 염 & $\frac{z}{s}$ & & & & & & & & & & & 8 \\
\hline $\mathbb{Z}$ & $\geqq$ & & & & & & & & & & & & \\
\hline$\frac{z}{x}$ & & & & & & & & & & & & & \\
\hline
\end{tabular}


En relación a las variables actitudinales, se ha encontrado una correlación muy alta $(0,50)$ entre la actitud hacia la lengua española y la actitud hacia los hispanohablantes. Al tratarse de una correlación positiva, el alumnado que refiere una actitud favorable hacia el español suele desarrollar también una actitud favorable hacia las personas de habla hispana.

En cuanto a las variables motivacionales, hay que remarcar que el grado de motivación general presenta una correlación positiva con los tres tipos de motivaciones estudiadas, hallándose una correlación muy alta con la motivación intrínseca $(0,59)$ y casi perfecta en el caso de la motivación instrumental $(0,84)$ y la motivación integradora $(0,82)$.

De igual modo, hay que remarcar que la motivación instrumental se relaciona positivamente con la motivación integradora con un coeficiente de 0,55. Se recuerda que las motivaciones no son excluyentes sino que se complementan. De esta forma, el alumnado motivado de forma instrumental lo estaría a su vez de forma integradora. Vemos que ambas motivaciones extrínsecas suelen coincidir en el mismo sujeto.

\section{Conclusiones}

De los resultados obtenidos en torno a las variables motivacionales se concluye que el grado de motivación global del alumnado de la sección internacional de español estudiada es alto (véase tabla 1). El aprendizaje del español como lengua extranjera por parte de este alumnado, por tanto, viene empujado por un alto deseo, interés y esfuerzo. Tras tal intensidad en la motivación, se esconde un deseo interno independiente de los logros externos (una motivación intrínseca) y/o una aspiración para conseguir otros fines (una motivación extrínseca: instrumental o integradora).

La motivación dominante para aprender el español es la intrínseca seguida de la instrumental (véase gráfica 2). El hecho de que la motivación dominante sea la intrínseca se podría explicar por la elección de cursar esta sección internacional de español de forma voluntaria. Por otra parte, la motivación instrumental podría deberse posiblemente al contexto preuniversitario ya que el aprendizaje del español beneficiará a los estudiantes para viajar y les proporcionará un aporte en su perfil profesional de cara a encontrar empleo en el mercado laboral nacional o internacional.

El análisis estadístico revela que la presencia de uno de los tres tipos de motivación analizados (intrínseca, instrumental e integradora) eleva el grado de motivación global, dadas la correlación casi perfecta entre motivación general y la motivación integradora o la instrumental (ambas extrínsecas) y la correlación muy alta entre motivación general y motivación intrínseca. Destaca la correlación casi perfecta encontrada, que demuestra que a mayor motivación extrínseca (instrumental o integradora), mayor será la motivación general que tenga el alumno. Ello significa que, en dicha muestra, poseer una motivación extrínseca intensifica el grado de motivación global más que la motivación intrínseca. ¿Podríamos generalizar que el grado de motivación global de los aprendientes de una LE se eleva más cuando la motivación extrínseca es mayor que la intrínseca? No necesariamente, investigaciones como la de Rodríguez-Lifante (2005) ponen de 
manifiesto un alto grado de motivación con predominio de la de carácter intrínseco.

Igualmente, es relevante la escasa relación existente entre la motivación general y la actitud hacia las lenguas extranjeras. Lo mismo ocurre en el caso de las motivaciones intrínseca, instrumental e integradora. No existe prácticamente una relación lineal entre la actitud manifestada hacia las lenguas extranjeras y la motivación. Lo cual sorprende dado el alto grado de motivación del alumnado y la excelente o muy buena actitud manifestada hacia las lenguas extranjeras, en su mayoría. Recordamos que estos resultados se ciñen a una muestra no representativa de una población.

Respecto a la motivación intrínseca, hemos de preguntamos cómo es posible que la relación lineal entre la motivación intrínseca de los aprendientes de ELE y la actitud que manifiestan hacia esta lengua sea nula (0). Esto significa que tener una motivación intrínseca mayor no se corresponde con manifestar una mejor actitud hacia la lengua española. La relación, de existir, no es de tipo lineal. Sin embargo, esta relación pasa a existir, eso sí, de forma baja, en el caso de la motivación extrínseca (instrumental e integradora). Igualmente, llama enormemente la atención la baja correlación entre la motivación intrínseca y las actitudes hacia la cultura hispana y los hispanohablantes. Por lo que el hecho de que los aprendientes estén motivados de forma intrínseca no implica necesariamente que generen una actitud favorable hacia la lengua y cultura estudiadas, y hacia los hablantes de la misma. Y en este punto se abre una línea de investigación que esclarezca con exactitud la relación entre las variables actitudinales hacia la lengua, cultura y hablantes, y la motivación intrínseca. Sin embargo, no es sencillo identificar el papel exacto de las variables afectivas en el aprendizaje de una lengua extranjera, dado que, como afirma Brown (1987), la cultura establece para cada persona un contexto de comportamiento afectivo y cognitivo. Y aquí hemos de añadir todas las características individuales o ambientales del proceso de aprendizaje, que varían en función del aprendiente y el contexto. Este compendio de variables que influyen en la motivación para el aprendizaje de una lengua extranjera es amplio, entre ellas, las actitudes específicas, la naturaleza del programa lingüístico, la temporización del aprendizaje, los recursos didácticos, las actividades, el deseo de aprendizaje, el contacto directo con los elementos culturales, la participación, la edad, etc. (Gardner y Tremblay, 1994).

Y en contraste con lo dicho anteriormente, la motivación instrumental sí presenta una correlación media con la actitud hacia la lengua española, aunque baja hacia la cultura hispana y nula hacia los hispanohablantes. Vemos, en este estudio, que cuando se presenta una motivación impulsada por un fin utilitario, la actitud hacia el español se incrementa, quizás por visión de la lengua como instrumento para alcanzar un fin, pero no la actitud hacia la cultura y hacia los hablantes. ¿Puede esto tener relación con la concepción de los aprendientes sobre el papel de la cultura en el aprendizaje de la LE? ¿AAcaso la perciben como prescindible, en contraste con la utilidad de la lengua? ¿̇mplica ello que han estudiado la lenguacultura de una forma fragmentada?

En cuanto a la motivación integradora, recordamos que esta presenta un carácter sociocultural y, sin embargo, las correlaciones con las actitudes hacia la 
lengua, cultura e hispanohablantes son bajas o casi nulas. Parece que, querer comprender, conocer o integrarse en una comunidad no conlleva la presencia de una actitud favorable hacia los integrantes de esta comunidad, su cultura y su lengua. ¿Sería esto extrapolable a otros contextos?

En relación a las variables actitudinales, se puede inferir de los datos recabados que el total de las actitudes estudiadas se valoran como muy buenas, resaltando como excelente la actitud hacia la lengua española y hacia los hispanohablantes. Es decir, el informante medio manifiesta actitudes favorables hacia los diferentes factores estudiados. De hecho, presenta actitudes muy buenas hacia: sí mismo, el profesorado, el grupo-clase, las asignaturas en español, el entorno de aprendizaje, las lenguas extranjeras y la cultura hispana. Igualmente, a la luz del análisis de los datos aportados por los informantes en el apartado 6, destaca la muy alta correlación entre actitud hacia la lengua española y la actitud hacia los hispanohablantes, que, además de ser excelentes, van de la mano, pues el alumnado que muestra una actitud excelente hacia los hispanohablantes también lo hace para el estudio de la misma lengua y viceversa. Esto contrasta con la correlación media entre la actitud hacia la lengua española y la actitud hacia la cultura hispana o entre la actitud hacia los hispanohablantes y la actitud hacia la cultura.

Por otro lado, resulta muy relevante que, dentro de las actitudes hacia la situación de aprendizaje (docente, entorno, grupo y asignatura), la actitud hacia el docente (quien cuenta con posibilidades suficientes para determinar los materiales, el clima de aula y programar la asignatura) casi no se correlaciona con las actitudes hacia el grupo, el entorno y las asignaturas. ¿̇Significa ello que la influencia del docente en el entorno, grupo o asignatura, podría ser limitada? Tal y como advierte Gardner (1972) las acciones del profesor pueden resultar infructuosas, frente a otras muchas influencias que actúan sobre ellos.

En definitiva, este trabajo nos plantea serias cuestiones sobre la relación entre la actitud hacia el profesorado y el resto de actitudes relativas a la situación de aprendizaje; la nula relación entre la motivación intrínseca y hacia la lengua española; o la motivación integradora y su baja o nula relación con las actitudes hacia la lengua, cultura e hispanohablantes. Tales preguntas nos plantean la necesidad de investigar a gran escala con muestras representativas, que nos permitan generalizar y delimitar la relación entre las variables afectivas (motivaciones y actitudes) que influyen a diario en el proceso de enseñanzaaprendizaje.

Nuestro cuestionamiento afecta también a la relación existente entre variables afectivas diferentes, pero relacionadas por diversos autores como Ezpí y Azurmendi (1996), al no encontrar correlación alta entre ninguna variable motivacional y actitudinal. Sin lugar a dudas, esto abre futuras líneas de investigación, que contemplen una muestra representativa, que estudien el papel de las actitudes en el constructo multidimensional del concepto de motivación. Esto nos sugiere un nuevo estudio que indague en las siguientes cuestiones żla existencia de motivación implica una actitud favorable? ¿ La presencia de una actitud favorable conlleva a la aparición de motivación, sea cual sea el tipo? Resulta trascendente averiguar si nuestros alumnos que exhiben una actitud favorable están realmente motivados 
para el aprendizaje del idioma. Dicha actitud favorable podría responder a la predisposición al docente, al grupo, al aula, e incluso a la lengua y la cultura extranjera, pero no necesariamente se vería acompañada de un deseo, del esfuerzo o el interés por aprender. Del mismo modo, podríamos cuestionarnos si la motivación nacida por el deseo interno despierta una actitud favorable hacia el objeto de aprendizaje.

\section{Agradecimientos}

Gracias al respaldo de la comunidad educativa se ha podido efectuar este estudio. Se agradece enormemente a la Consejería de Educación en Polonia su consentimiento para realizar esta investigación, y al alumnado y profesorado del Liceum XXXII Halina Poświatowska su disposición a participar en la misma.

\section{Bibliografía}

ARNAL, J., D. DEL RINCÓN \& A. LATORRE (1992): Investigación educativa. Metodologías de investigación educativa. Barcelona: Labor.

BACHMAN, L. F. (2004): Statistical Analyses for Language Assesment. Cambridge: Cambridge University Press.

BROWN, H. D. (1973): "Affective variables in second language acquisition". Language Learning, 23, pp. 231-244.

COHEN, J. (1977): Statistical power analysis for the behavioral sciences. Nueva York: Academic Press.

DÖRNYEI, Z. (1994): "Motivation and motivating in the foreign language classroom". Modern Language Journal, 78 (3), pp. 273-284.

ESPÍ GUZMÁN, M.J. \& M.J. AZURMENDI AYERBE (1996): "Motivación, actitudes y aprendizaje en el español como lengua extranjera". Revista española de lingüística aplicada, (11), pp. 63-76.

GARDNER, R. C. (1985): Social Psychology and Second Language Learning: The role of Attitudes and Motivation. Londres: Edward Arnold.

GARDNER, R. C. \& W. LAMBERT (1972): Attitudes and Motivation in Second Language Learning. Londres: Newbury Honse.

GARDNER, R. C. \& P. F. TREMBLAY (1994): "On motivation, measurement and conceptual considerations". The Modern Language Journal, 78, (4), pp. 52427.

MADRID, D. (1999): La investigación de los factores motivacionales en el aula de idiomas. Granada: Grupo Editorial Universitario.

MINERA REYNA, L. E. (2010): "El cuestionario (MAALE), técnica para recolección de datos sobre las variables afectivas motivación y actitudes en el aprendizaje de una lengua extranjera". Revista Electrónica RedELE, n¹8, junio de 2010.

TRAGANT, E. \& C. MUÑOZ (2000): La motivación y su relación con la edad en un contexto escolar de aprendizaje de una lengua extranjera. Segundas lenguas: adquisición en el aula. C. MUÑOZ (ed.). Barcelona; Ariel Lingüística.

España. Real Decreto 1027/1993, de 25 de junio, por el que se regula la acción educativa en el exterior. Boletín oficial del estado, 6 de agosto de 1993, núm. 187, pp. 23941-23948. 
RODRÍGUEZ-LIFANTE, A. (2015): Motivación y actitudes como variables afectivas en aprendices griegos de español como lengua extranjera. Alicante: Universidad de Alicante.

SPOLSKY, B. (1989): Conditions for second language learning: introduction to a general theory. Oxford: Oxford University Press.

SPYCHAŁA-WAWRZYNIAK, M. \& L. SAGERMANN (2016): "La motivación de los alumnos polacos para el aprendizaje del español como lengua extranjera". Studia Romanica Posnaniensia, 43 (3), pp. 85-102. 